\title{
Tracer Mixing at Fracture Intersections
}

\section{Guomin Li}

\author{
Earth Sciences Division \\ Lawrence Berkeley National Laboratory \\ One Cyclotron Road, Berkeley, California
}

\begin{abstract}
Discrete network models are one of the approaches used to simulate a dissolved contaminant, which is usually represented as a tracer in modeling studies, in fractured rocks. The discrete models include large numbers of individual fractures within the network structure, with flow and transport described on the scale of an individual fracture. Numerical simulations for the mixing characteristics and transfer probabilities of a tracer through a fracture intersection are performed for this study. A random-walk, particle-tracking model is applied to simulate tracer transport in fracture intersections by moving particles through space using individual advective and diffusive steps. The simulation results are compared with existing numerical and analytical solutions for a continuous intersection over a wide range of Peclet numbers. This study attempts to characterize the relative concentration at the outflow branches for a continuous intersection with different flow fields. The simulation results demonstrate that the mixing characteristics at the fracture intersections are a function not only of the Peclet number but also of the flow field pattern.
\end{abstract}




\section{INTRODUCTION}

Fractures represent preferential pathways along which a dissolved contaminant, which is usually represented as a tracer in modeling studies, can migrate rapidly in geologic formations. Discrete network models are one of the approaches used to simulate tracer transport in fractured rocks. The discrete models include large numbers of individual fractures within a network structure, with flow and transport described on the scale of an individual fracture and from fractures to fractures. What is not sufficient clear for tracer transport in discrete fracture networks is how various tracer transfer processes, which act on a number of different scales, interact to determine transport patterns and tracer concentrations, and how we can develop quantitative methods to describe transport in a rock mass where fractures provide the dominant pathways for transport migration (Smith and Schwartz 1993). Our particular issue is what is the flow and transport pattern at fracture intersections.

There are basically two types of fracture intersections (or junctions) formed when one fracture crosses a second fracture: continuous intersections and discontinuous intersections. A continuous intersection occurs when each inflow branch is connected by a corresponding outflow branch. At a discontinuous intersection, the sequence of inflow branches is interrupted by one or more outflow branches (an example is a T-intersection) (Berkowitz and others 1994).

Wilson and Witherspoon (1976) describe experimental studies of flow through a continuous intersection; they proposed a streamline routing theory, in which the mass 
flux is determined only by the discharge patterns in related fractures. Hull and Koslow (1986) report laboratory experiments for both continuous and discontinuous intersections, and explore streamline routing to explain the mass transport through the intersections. Robinson and Gale (1990) provide examples that illustrate the differences in mass distribution that develop with two different approximations: streamline routing and complete mixing in the fracture intersection. In the fracture network models, there can be significantly greater transverse spreading of tracer under the assumption of complete mixing, while streamline routing tends to minimize transverse spreading. Philip (1988) has solved the boundary-value problem that describes the micro-scale flow pattern at an intersection of two equal-aperture orthogonal fractures. Philip (1988) characterizes the mixing process at a fracture intersection in terms of a local Peclet number, representing the interplay between advective and diffusive tracer transfer. Park and Lee (1999) provide simple analytical solutions for the mixing characteristics at the continuous fracture intersections. As the Peclet number increases, the analytical solutions also indicate the transition from complete mixing to streamline routing at a fracture intersection (Park and Lee 1999).

The particle-tracking technique has been widely used to study the solute dispersion in a heterogeneous porous medium. It was also used by Schwartz and others (1983) to address the dispersion in an idealized fractured medium consisting of two sets of orthogonal fractures. Berkowitz and others (1994) applied a random-walk particle-tracking method to study mixing behavior at an idealized fracture junction. In their studies, mixing ratios are expressed in terms of a local Peclet number. They indicate that as a general 
observation, the concept of complete mixing within a fracture intersection does not properly represent the mass transfer process at any value of the Peclet number. Li (unpub. 1995) applied a numerical lattice-gas automata (LGA) model to study the relationship between mixing behavior and the local Peclet number. The LGA simulations of the mixing behavior at fracture intersections predict that for Peclet numbers smaller than 1 , diffusion dominates the process of tracer transport, and complete mixing occurs. For Peclet numbers larger than 1, both diffusion and advection play important roles in the mixing process. Stockman and others (1997) applied LGA and lattice Boltzmann (LB) methods to simulate the mixing ratio versus the Peclet number, and compared their results with other experiments and numerical simulations. They investigated the significant effect of the boundary conditions and size of the computational domain on the result observed. Results from the LGA and LB simulations and the simulations of Berkowitz and others (1994) shows significant differences from each other (Stockman and others 1997).

The objective of our current study is to conduct numerical simulations using the randomwalk particle-tracking method and to investigate the mixing behavior of tracer transport at fracture intersections, and to compare the results with those for earlier studies presented above. An equal flow rate model, in which the flow rate is the same in all fracture branches, is used as our base model for simulating the mixing behavior for four scenarios representing hydrodynamic dispersion: pure molecular diffusion, mechanical dispersion and two combinations of molecular diffusion and mechanical dispersion. The pure molecular model is also used to investigate the effect of initial tracer concentration. 
Two non-equal flow rate models are used to investigate the effect of changing flow fields on the mixing behavior at fracture intersections. Finally, comparisons with existing numerical and analytical solutions are discussed.

\section{METHODOLGY}

A two-dimensional inviscid and irrotational steady flow is assumed in this study (Figure 1). The pressure $\mathrm{H}$ in the domain is described by the differential equation

$$
\nabla^{2} \mathrm{H}=0
$$

subject to boundary conditions.

The fluid velocity can be defined for a chosen volumetric flow through the fracture intersection under chosen boundary conditions, for different permeability values of the inflow and out-flow branches. Then, the advective transfer of tracer spreading (resulting from streamlines taking a two-dimensional configuration with differing path lengths controlled under the distribution of velocities) can be estimated.

To compare different numerical results, we introduced the Peclet number $\mathrm{P}_{\mathrm{e}}$ to represent the flow conditions. The local Peclet number can be defined as

$$
\mathrm{P}_{\mathrm{e}}=1.414 \mathrm{bv} / \mathrm{D}
$$


where $\mathrm{v}(\mu \mathrm{m} / \mathrm{s})$ is the average velocity within the intersecting area (Figure 1$), \mathrm{b}(\mu \mathrm{m})$ is the width of the fractures (where two intersecting fractures are assumed to have the same width), and $\mathrm{D}\left(\mu \mathrm{m}^{2} / \mathrm{s}\right)$ is the coefficient of local hydrodynamic dispersion. The coefficient of local hydrodynamic dispersion is defined as the sum of the coefficients of mechanical dispersion and molecular diffusion. The local Peclet number can be approximated by

$$
\mathrm{P}_{\mathrm{e}}=1.414 \mathrm{bv} /\left(\alpha_{\mathrm{L}} \mathrm{v}+\mathrm{D}^{\prime}\right)
$$

where $\alpha_{L}(\mu \mathrm{m})$ is the coefficient of longitudinal dispersion, and $D^{\prime}\left(\mu \mathrm{m}^{2} / \mathrm{s}\right)$ is the coefficient of molecular diffusion. The Peclet number expresses the relative importance between advection and diffusion within the intersection area. As the fluid velocity increases, the Peclet number increases, and the influence of diffusion decreases. On the other hand, as the fluid velocity decreases, the Peclet number decreases, and diffusion tends to play a relatively more important role in the transport process.

Diffusive processes within the individual fracture will depend upon boundary conditions, permeability distributions, residence time of mass in the system, and the magnitude of the fluid diffusion coefficient. Clearly, if the residence time in the fracture is sufficiently long, diffusion spreads mass across streamlines and results in a transverse concentration profile.

The general nonreactive mass-transport problem for a dissolved, neutrally buoyant species involves the solution of the mass balance equation 


$$
\partial \mathrm{c} / \partial \mathrm{t}+\nabla(\mathrm{c} \bullet \mathrm{v})-\nabla(\mathrm{D} \bullet \nabla \mathrm{c})=0
$$

for the concentration c over a period of time, subject to a set of initial and boundary conditions for $\mathrm{c} . \mathrm{D}\left(\mu \mathrm{m}^{2} / \mathrm{s}\right)$ represents the dispersion coefficient.

In the calculations that follow, a random-walk, particle-tracking model is applied to simulate tracer transport in fracture intersections by moving particles through space using individual advective and diffusive steps. This method is based upon analogs between mass transport equations and certain stochastic differential equations. A particle is displaced according to the following simple relationship (Tomspon and Gelhar 1990):

$$
\mathrm{X}^{\mathrm{n}}=\mathrm{X}^{\mathrm{n}-1}+\mathrm{A}\left(\mathrm{X}^{\mathrm{n}-1}\right) \Delta \mathrm{t}+\mathrm{B}\left(\mathrm{X}^{\mathrm{n}-1}\right) \cdot \mathrm{Z} \sqrt{ } \Delta \mathrm{t}
$$

where $\mathrm{X}^{\mathrm{n}}$ is its position at time level $\mathrm{n} \Delta \mathrm{t}, \mathrm{A}$ is a deterministic velocity vector, $\mathrm{B}$ is a deterministic scaling matrix, and $\mathrm{Z}$ is a vector of random numbers with a mean of zero and variance of one. The motion of one particle will thus be statistically independent from that of another. If a large number of identical particles associated with a particular component are moved simultaneously, then their number density $f(x, t)$ will approximately satisfy the Ito-Fokker-Planck equation (Kinzelbach 1988):

$$
\partial \mathrm{f} \partial \mathrm{t}+\nabla(\mathrm{A} \bullet \mathrm{f})-\nabla \nabla:\left(1 / 2 \mathrm{~B} \bullet \mathrm{B}^{\mathrm{T}} \bullet \mathrm{f}\right)=0
$$


Equation (4) represents the mass balance for a conservative aqueous constituent. The particle-tracking method succeeds if the particle number density $\mathrm{f}$ in Equation (6) is proportional to $\mathrm{c}$ in Equation (4), subject to A and B by

$$
\mathrm{A} \equiv \mathrm{v}+\nabla \bullet \mathrm{D}
$$

and

$$
\mathrm{B} \bullet \mathrm{B}^{\mathrm{T}} \equiv 2 \mathrm{D}
$$

Tompson and Gelhar (1990) discussed some of the issues concerning the computational approximations required in applying a random-walk particle-tracking model (Equation 5).

\section{MODEL STRUCTURE AND BOUNDARY CONDITIONS}

Figure 1 shows the fracture intersection model and its boundary conditions. The groundwater flow through this domain is also calculated for constant piezometric head boundaries: the left-hand and bottom boundaries are assumed to be at $1 \mu \mathrm{m}$ head, and the right-hand and top boundaries, which are $70 \mu \mathrm{m}$ on the opposite sides respectively, are assumed to be at $0 \mu \mathrm{m}$ head. The flow rates into left and bottom branches are assumed to be equal. .

The 2-D finite element method is used to discretize the flow domain $(70 \times 70$ elements; each with a dimension of $1 \times 1 \mu \mathrm{m}$ ). The domain is divided into two intersecting fractures, each $10 \mu \mathrm{m}$ wide, and with a permeability of $1.0 \mu \mathrm{m}^{2}$, surrounded by a very

low-permeability background of $1.0 \times 10^{-30} \mu^{2}$, representing an impermeable rock 
matrix (Figure 1). The permeability is uniform over the flow field; i.e, the flow-rate in the left fracture equals that in the lower fracture (flow ratio 50/50). Fractures (the higher permeability domain) represent preferential pathways along which a solute can migrate rapidly; however, tracer diffusion from the fractures to the matrix (the lower-permeability domain) can significantly reduce migration rates along the fractures.

For our study, particles are introduced at the left-hand high-head boundary. The randomwalk method is based on particle transport under the influence of both rock spatial fluid velocities and diffusion. It is possible for some particles to travel backward across the left-hand inflow boundary or to jump into the impermeable rock matrix from one time step to the next. We assume that the particle will disappear if it goes out the left-hand boundary, or will be bounced back (perfect reflection) into the modeling domain if it goes out of the fracture domain into the low-permeability background region. This confines the tracer transport, represented by a number of particles, to the fractures and the fracture intersection.

\section{SIMULATION RESULTS}

From the flow model, the velocities can be calculated at any position in the domain. Five thousand particles are introduced at a distance of $5 \mu \mathrm{m}$ from the left-hand highpiezometric head boundary and are collected at the right-hand and top low-piezometric head boundaries. A plot of the number of particles collected at the right-hand boundary and top boundary at different arrival times constitutes the breakthrough curves. In these 
plots, only 20 particles were used to show the solute flow lines and 5,000 particles to plot the breakthrough curves.

The mixing rules at fracture intersections applied in numerical simulations of mass transport in modeling studies of discrete fracture networks are usually of three types: streamline routing, streamline routing with diffusion within fracture intersections, and complete mixing (Smith and Schwartz 1993). Streamline routing and complete mixing rules may be appropriate for very high and very low Peclet numbers, respectively. However, within a fracture network, local velocities vary greatly and Peclet numbers are expected to take on a wide range of values. Therefore, streamline routing with diffusion within fracture intersections may be realistic to simulate tracer transport in discrete fracture networks. In this study, numerical simulations are performed for a wide range of Peclet numbers, between $5 \times 10^{-3}$ and $6 \times 10^{4}$, to study the validity of the various assumptions.

As previously stated, the Peclet number (Equation 3) is a function of longitudinal dispersion and molecular diffusion. Bear (1979) and Fried (1971) have plotted the results of many experiments that show the relationship between molecular diffusion and hydrodynamic dispersion. Experimental results with low Peclet numbers indicate five ranges: 1) $\mathrm{P}_{\mathrm{e}} \leq 0.4$, in which molecular diffusion predominates, as the average flow velocity is very small; 2) $0.4<\mathrm{P}_{\mathrm{e}} \leq 5$, in which the effects of mechanical dispersion and molecular diffusion are of the same order of magnitude; 3) $5<\mathrm{P}_{\mathrm{e}} \leq 300$, in which the spreading is mainly by mechanical dispersion; 4) $300<\mathrm{P}_{\mathrm{e}} \leq 30,000$, in which mechanical 
dispersion dominates and the effect of molecular diffusion is negligible; and 5) $\mathrm{P}_{\mathrm{e}} \geq$ 30,000 , in which pure mechanical dispersion occurs, but beyond the range of Darcy's law.

Based on the above discussion, four models are used in our simulations to represent the relationship between molecular diffusion and longitudinal dispersion: pure molecular diffusion, mechanical dispersion and two combinations of molecular diffusion and mechanical dispersion.

In the pure molecular diffusion and pure mechanical dispersion models, Equation (3) is simplified as $\mathrm{P}_{\mathrm{e}}=1.414 \mathrm{bv} / \mathrm{D}^{\prime}$ and $\mathrm{P}_{\mathrm{e}}=1.414 \mathrm{~b} / \alpha_{\mathrm{L}}$, respectively. In the pure molecular model, we consider only molecular diffusion, without advection by mechanical dispersion, even for high flow velocities (high Peclet numbers). The mixing may be accelerated and overestimated by the diffusion due to the difference between the concentrations of the streamlines. In the pure mechanical model, we consider only advection by longitudinal dispersion even for low velocities (low Peclet numbers). Therefore the mixing may be decreased and underestimated. These two models may provide the range of the mixing ratio in fracture intersections over a wide range of Peclet numbers.

\subsection{Mixing Process}

Figure 2 shows the 20 particle traces with different Peclet numbers for the base model. The pure molecular model is used to simulate the particle movement in the fractures and their intersection. 
Figure 2a shows that all the particles move from the left-hand boundary to the top boundary at a very high Peclet number of $1.18 \times 10^{4}$. This indicates that the diffusion term is too small to affect particle movement in the flow field, so those particles follow the streamlines. This is an example of streamline routing. In Figure $2 b$, under the conditions of a Peclet number of 118, some of the particles jump into nearby streamlines; some then move out of the right-hand boundary.

We need to know what percentage of particles can go through the right-hand boundary, and whether complete mixing can happen. It is clear that the smaller the Peclet number, the higher the number of particles that will go through the right-hand boundary.

Figure 3 shows the relative concentration of particle distribution in the outflow branches for a range of Peclet numbers between $5 \times 10^{-3}$ and $6 \times 10^{4}$. It is clear in Figure 3 that solutions using the pure mechanical dispersion model underestimate the mixing ratio when compared with the results of the pure molecular diffusion model. As the Peclet number decreases, both results show more mass mixing at the intersection, and the mixing ratio increases toward an asymptotic value of 0.5 , indicating that complete mixing may be occurring. Both models show a declining mixing ratio at the intersection as the Peclet number decreases toward zero. The results of the two combinations with ratios of longitudinal dispersion to molecular diffusion, $50: 50$ and $99: 1$, fall between the results from the pure molecular diffusion and the pure mechanical models. The significantly lower results from the pure mechanical model indicate that the mixing ratio at the fracture 
intersection should be higher in the pure molecular diffusion model than in the pure mechanical model for the same Peclet number. Therefore, mixing accelerated by diffusion due to the difference in concentrations of the streamlines must be included to be more realistic. The results of the pure mechanical model and the pure molecular model provide a wide range of mixing ratios for a range of Peclet numbers.

\subsection{Effect of Initial Tracer Input Location}

To investigate the relationship between the initial position of the particles relative to the left-hand boundary and the resulting characteristics of tracer transport, the particles are introduced 25, 10 and $5 \mu \mathrm{m}$ from the intersection in the left-hand high-piezometric head branch. In these calculations, 5,000 particles are used in the pure molecular diffusion model to track the tracer transport and to plot the breakthrough curves. The flow rate in the left-hand fracture branch is assumed to be same as that in the lower fracture branch (flow ratio 50/50).

As seen in Figure 4, when $\mathrm{P}_{\mathrm{e}}<1$ and $\mathrm{P}_{\mathrm{e}}>10$, for the model with particles introduced 5 $\mu \mathrm{m}$ from the fracture intersection, relative concentrations from the right fracture branch are slightly higher than those from the other two models. However, for the $10 \mu \mathrm{m}$ model (particles introduced $10 \mu \mathrm{m}$ from the fracture intersection; i.e., the width of the fracture), no significant difference is found even at the lower Peclet number when compared to the $25 \mu \mathrm{m}$ model. This indicates that errors due to the initial tracer concentration in the left 
fracture branch can be ignored when the particles are placed $10 \mu \mathrm{m}$ or more from the fracture intersection.

\subsection{Effect of Flow-Rate Ratio}

In the above models, permeability, and therefore flow rate, are the same in all fracture branches. The flow field is expressed in terms of the ratio of the inflow in the left branch to that in the lower fracture branch (flow ratio 50/50).

If we adjust the permeability distribution, we will obtain different ratios of the flow rate in the right-hand fracture branch and the upper fracture branch. Two models, Model A and Model B, are chosen to study the tracer mixing in the fracture intersection. In Model A, we assume that the permeability in the upper and lower fracture branches is two times that in the left and right fracture branches. The permeability in the intersection area is the same as those in both left and right fracture branches. The ratio of the flow rate in the right fracture $\left(\mathrm{Q}_{\mathrm{e}}\right)$ to that in the lower fracture $\left(\mathrm{Q}_{\mathrm{n}}\right)$ is about $35 / 65\left(\mathrm{Q}_{\mathrm{e}} / \mathrm{Q}_{\mathrm{n}}\right)$. In Model $\mathrm{B}$, it is assumed that the permeability in the left and right fracture branches is 10 times that of the other two fracture branches, including the intersection area. The ratio of the flow rate in the left-hand fracture branch to that in the lower fracture branch is around $83 / 17$ $\left(Q_{e} / Q_{n}\right)$.

In Models A and B, all particles are introduced $25 \mu \mathrm{m}$ from the fracture intersection in the left-hand high-piezometric fracture branch, and are collected at the right-hand low head boundary. In these calculations, 20 particles are used to show the solute flow lines 
and 5,000 particles to plot the breakthrough curves. Pure molecular diffusion is applied to simulate the mixing behavior at fracture intersections.

As shown in Figure 5a, for Model A, all particles move from the left-hand boundary to the top boundary at a very high Peclet number of $1.18 \times 10^{4}$. The mass distribution is controlled by the function of streamlines. Figures $5 \mathrm{~b}$ shows that some of the incoming particles occupy the whole upper fracture and the rest of the particles from the left-hand fracture branch flow into the right-hand branch.

Figure 6 shows the relative concentration of particle distribution in the outflow branches versus the Peclet number in Model A, Model B and the equal-flow-rate pure molecular model $\left(\mathrm{Q}_{\mathrm{e}} / \mathrm{Q}_{\mathrm{n}}=35 / 65,83 / 17\right.$ and 50/50, respectively). It is clear in Figure 6 that the relative concentration is not only affected by the mixing processes but also controlled by the flow fields.

In the right fracture branch, the mixing relative concentration of the models is near 0.5 ; i.e., complete mixing, at low Peclet numbers (Figure 6a). Streamline routing might occur at somewhere above a Peclet number of 500. Due to the relatively low flow rate through the right-hand branch in Model A, the particles easily go through the top fracture branch. Therefore, the result from Model A is an underestimation when compared with the base model. On the other hand, Model B has a relatively high flow rate through the right-hand fracture branch. Therefore, the relatively high-concentration number associated with the 
configuration of the flow field $\left(\mathrm{Q}_{e} / \mathrm{Q}_{\mathrm{n}}=83 / 17\right)$ remains constant as the Peclet number increases toward a higher value than 1 (Figure 6a).

Mixing characteristics in terms of the resulting percentage of the relative concentration from the left fracture branch travelling into the top fracture branch at fracture intersections in Model A and Model B are compared with the base model $\left(\mathrm{Q}_{\mathrm{e}} / \mathrm{Q}_{\mathrm{n}}=50 / 50\right.$;

Figure 6b). At $\mathrm{P}_{\mathrm{e}}<10^{-2}$, the particles completely mix at the intersection and then dilute into the top fracture branch. For Models A and B, the transition zones have a range of about 3 orders of magnitude ( $10^{-2}$ to 10$)$. We expect that as Peclet numbers increase above 10, tracer transport is dominated by streamline routing, and the relative concentration in the top fracture branch is controlled by the flow fields configurations.

\section{COMPARISON WITH EARLIER STUDIES AND DISCUSSION}

We have compared our results with the numerical solutions of Berkowitz and others (1994) and Li (unpub.1995), and the analytical results of Park and Lee (1999). For the comparison, the tracer with relative concentration of 1 is introduced only into the left inflow branch (Figure 1). Results for an equal flow rate case are plotted in Figure 7, which shows that the results of the pure molecular diffusion model in this study match well the results of Li (unpub.1995) and Stockman and others (1997). Both the results of Berkowitz and others (1994) and Park and Lee (1999) underestimate the mixing ratio as compared with our results and those of Li (unpub.1995) and Stockman and others (1997). 
Berkowitz and others (1994) applied a random-walk particle-tracking method to study mixing characteristics at fracture intersection. They never observed diffusion-controlled complete mixing in a simulation, even at an intersection Peclet number as low as $3 \times 10^{-3}$. They explained this less-complete mixing by noting that particles entering the intersection on a streamline close to the left side of the wall of the left-hand fracture have a higher probability of moving across the dividing streamline into the left flow region than of remaining inside the original flow domain. Our results indicate that an error due to the initial tracer input location in the left fracture branch which can be ignored when the particles are introduced close to the fracture intersection.

Park and Lee (1999) applied simple analytical solutions for the mixing characteristics and transfer probabilities of mass at the fracture intersection. They indicated that possible underestimation of both their solution and that of Berkowitz and others (1994) compared with Stockman and others (1997) might be explained by the assumptions on the boundary conditions and the occurrence of longitudinal diffusion. They concluded that the boundary conditions at fracture walls might not be responsible for the underestimation of the mixing ratio, and also that the mixing ratio may be underestimated unless longitudinal diffusion is considered, especially at a low Peclet number.

Li (unpub.1995) and Stockman and others (1997) applied lattice gas automata (LGA) numerical simulations to investigate the mixing behavior at the fracture intersection. Their results shown that as the Peclet number decreases, the mixing ratio at the intersection increases toward an asymptotic value of 0.5 . When the Peclet number is 
smaller than 0.68 , there is complete mixing. They expect that at a Peclet number somewhere above $3 \times 10^{2}$, the mixing ratio will approach zero.

\section{SUMMARY}

The purpose of this study was to apply random-walk methods to simulate the mixing behavior of tracer transport at an idealized fracture intersection. Our results show that the Peclet number is the key parameter controlling tracer mixing at a fracture intersection. Due to difficulty in applying the unknown nonlinear relationship between mechanical dispersion and molecular diffusion, a pure mechanical dispersion (longitudinal dispersion) model, a pure molecular diffusion model and two linear combinations of mechanical dispersion and molecular diffusion were proposed to simulate the mixing behavior versus the Peclet number. These results provide the mixing characteristics over a range of Peclet numbers between $5 \times 10^{-3}$ and $6 \times 10^{4}$.

Complete mixing may happen as the Peclet number becomes less than 1 . The range of our results of the pure molecular diffusion model includes the results of Li (unpub.1995) and Stockman and others (1997; Figure 7). From these results we expect that a nonlinear combination of molecular diffusion and mechanical dispersion, i.e., a relatively high molecular diffusion coefficient at low Peclet numbers, and vice versa, may fit well with the realistic mixing process.

For an equal flow rate model with a very small Peclet number, the streamlines are no longer important for particle migration calculations. Complete mixing might occur, so 
that the relative concentration of tracer moving across the right-hand boundary is near 0.5 , as would be expected. As the Peclet number increases somewhere above $10^{2}$, the mixing ratio approaches zero, the tracer transport is dominated by the streamline routing, and the relative concentration in the right-hand branch approaches 0 . These results indicate a transition zone of about 3 orders of magnitude in Peclet numbers $\left(10^{-1}\right.$ to $\left.10^{2}\right)$.

Two non-equal flow rate models, Model $\mathrm{A}\left(\mathrm{Q}_{\mathrm{e}} / \mathrm{Q}_{\mathrm{n}}=35 / 65\right)$ and Model $\mathrm{B}\left(\mathrm{Q}_{\mathrm{e}} / \mathrm{Q}_{\mathrm{n}}=\right.$ 83/17), were used to investigate the effect of flow fields on the mixing behavior at fracture intersections. Under a low Peclet number of $10^{-2}$, the particles completely mix at the intersection and then dilute into outflow fracture branches. We expect that as Peclet numbers increase above some value over 10 , tracer transport is dominated by streamline routing. In the equal flow rate model, the transition zones exist. However, the transition zones have different ranges when compared with the equal flow rate model. For a nonequal flow rate model, our results indicate that the flow fields as well as the Peclet numbers control tracer transport.

\section{ACKNOWLEDGEMENTS}

The authors thank Chin-Fu Tsang and Mary Pratt of Lawrence Berkeley National Laboratory (LBNL) for their helpful comments and careful review of the manuscript. This work was supported by the Japan Nuclear Fuel Cycle Development Corporation (JNC) under a binational agreement between JNC and the U.S. Department of Energy, Office of Science, Office of Environmental Management, and performed at LBNL under Contract No. DE-AC03-76SF00098. 


\section{REFERENCES}

Bear J. (1979) Hydraulics of Groundwater. McGraw-Hill, New York.

Berkowitz B.C. and Naumann Smith L. (1994) Mass transfer at fracture intersections: An evaluation of mixing models. Water Resour. Res. 30: 1765-1773

Fried J. J. (1975) Groundwater Pollution. Elsevier, Amsterdam.

Hull L. C. and Koslow K. (1984) Streamline routing through fracture junctions. Water Resour. Res. 22: 1390-1400

Kinzelbach W. (1988) The random walk method in pollutant transport simulation. In Groundwater Flow and Quality Modeling. Edited by Custodio E., Gurgui A. and Lobo Ferreira J. P. D. Reidel, Norwell, Mass. 227-245

Park Y. J. and Lee K. K. (1999) Analytical solutions for solute transfer characteristics at continuous fracture junctions. Water Resour. Res. 35: 1531-1537

Philip J. (1988) The fluid mechanics of fracture and other junctions. Water Resour. Res. 24: $239-246$

Robinson J.W. and Gale J.E. (1990) A laboratory and numerical investigation of solute transport in discontinuous fracture systems. Ground Water 28: 25-36 
Schwartz F. W., Smith L. and Crowe A. S. (1983) A stochastic analysis of macroscopic dispersion in fracture media. Water Resour. Res. 19: 1253-1265

Smith L and Schwartz F. W. (1993) Solute transport through fracture networks. In: Flow and Contaminant Transport in Fractured Rock. Edited by Bear J., Tsang C. F. and Marsily G. Academic Press, San Diego, California. 129-167

Stockman H. W., Li C. and Wilson J. L. (1997) A lattice-gas and lattice Boltzmann study of mixing at continuous fracture junctions: Importance of boundary conditions. Geophys. Res. Lett. 24: 1515-1518

Tompson A. F. B. and Gelhar L. W. (1990)Numerical simulation of solute transport in three-dimensional, randomly heterogeneous parous media. Water Resour. Res. 26: 25412562

Tsang Y.W. and Tsang C.F. (1989) Flow channeling in a singer fracture as a twodimensional strongly heterogeneous porous medium. Water Resour. Res. 25: 2076-2080

Wilson C. R. and Witherspoon P. A. (1976) Flow interference effects at fracture intersections. Water Resour., Res. 12: 102-104 


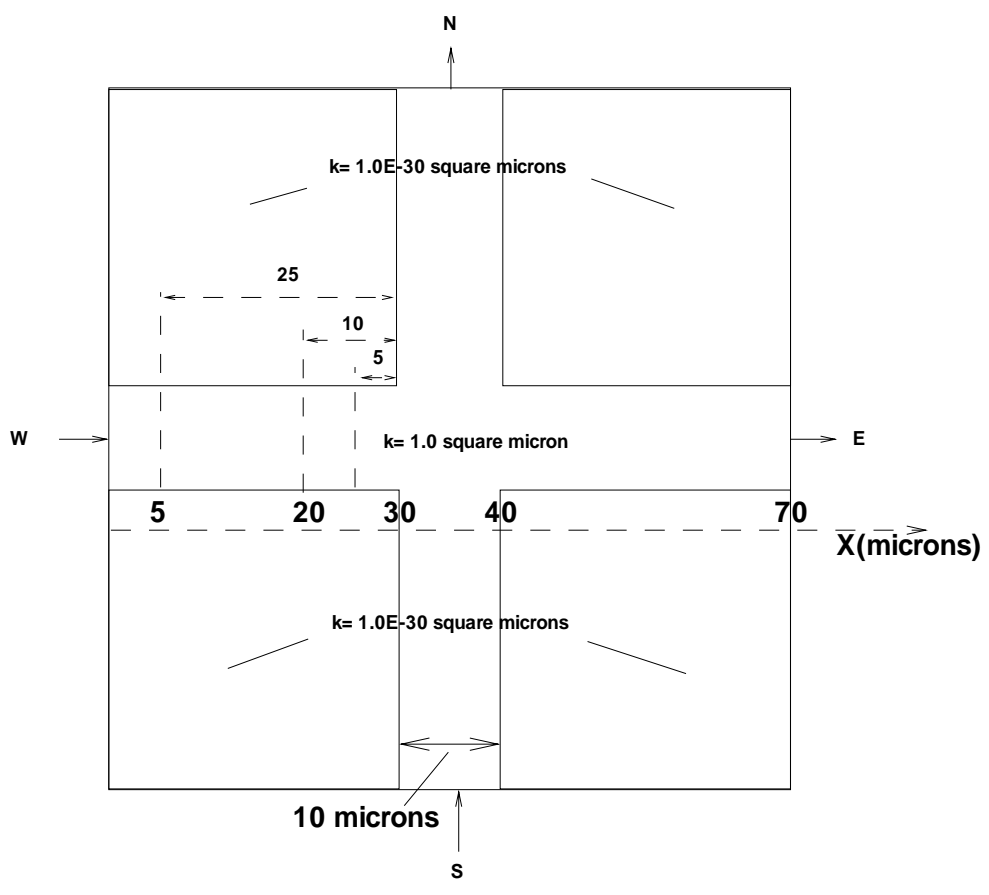

Figure 1. Fracture intersection model and boundary conditions. Pressure gradient from left to right and from down to up is $1.43 \times 10^{-4}$. 


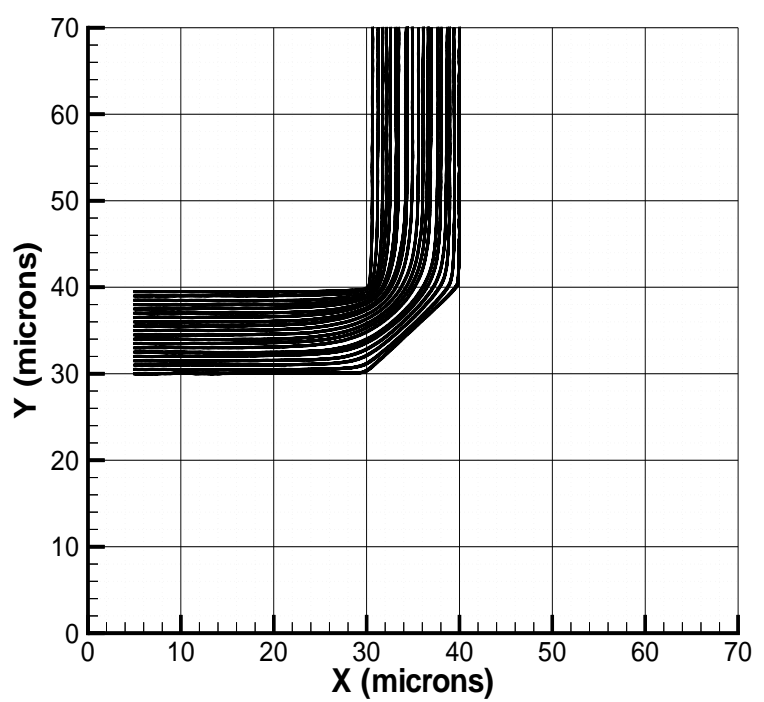

b

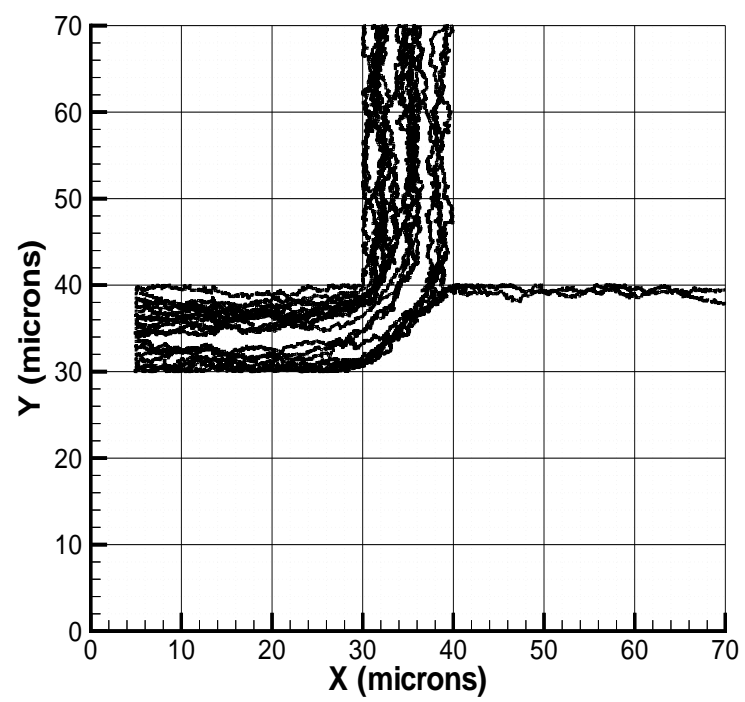

Figure 2. Spatial particle trace in the base model with flow rate ratio $Q_{e} / Q_{n}=50 / 50$ for (a) Peclet number $1.18 \times 10^{5}$ and (b) Peclet number $1.18 \times 10^{2}$. 


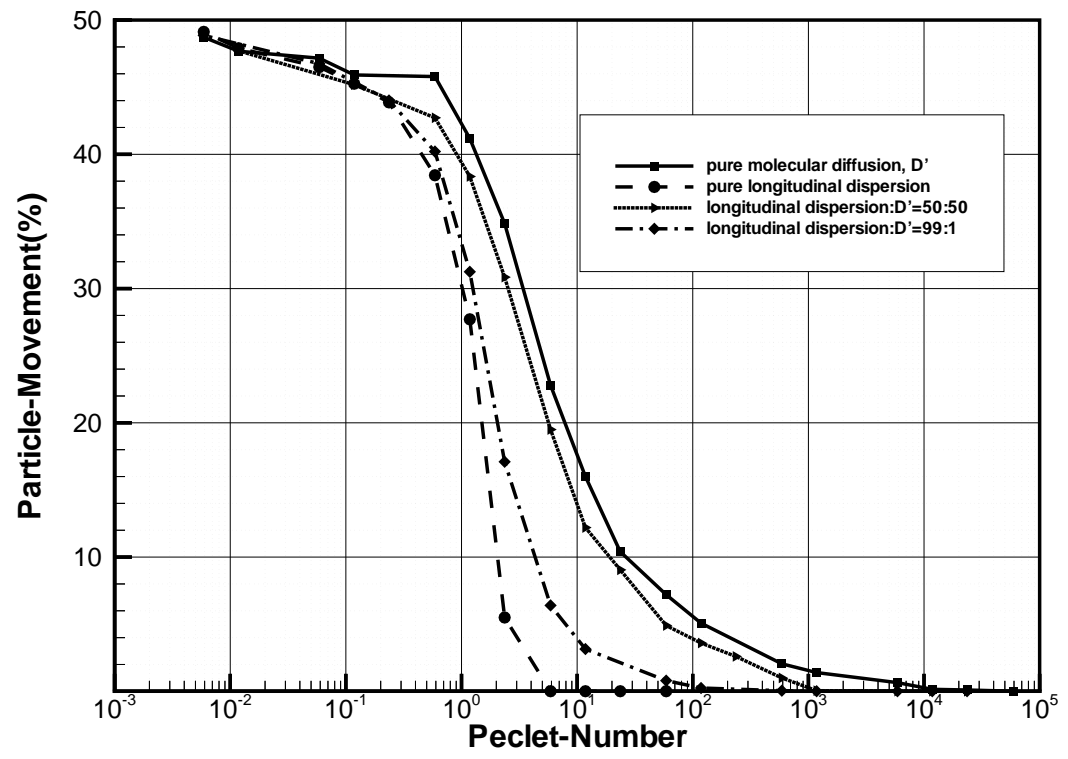

Figure 3. The relative concentration percentage of the particles passing through the boundaries over the total particles is a function of the Peclet number. The low curve is the percentage of the relative concentration passing through the right-hand boundary. Particles are placed in the left-hand fracture at $5 \mu \mathrm{m}$ from the left boundary. 


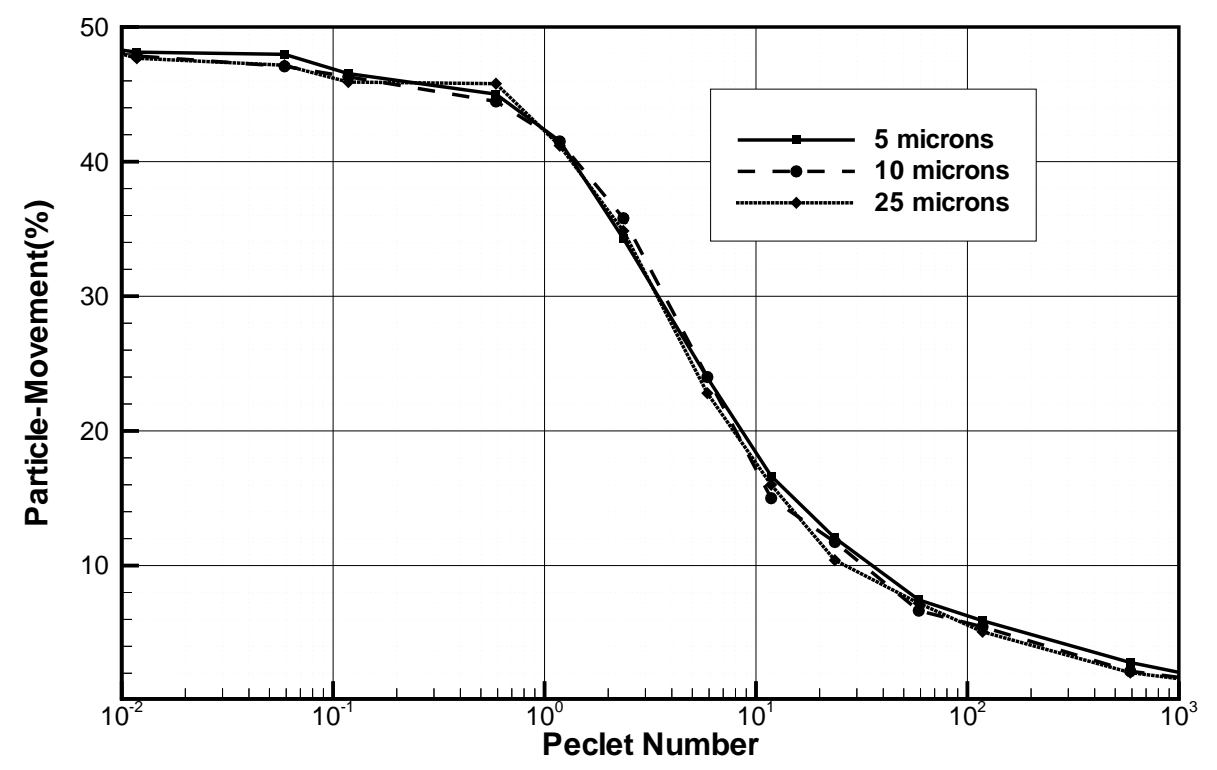

Figure 4. Comparisons of mixing characteristics at fracture intersections in terms of the resulting percentage of the relative concentration from the left fracture branch travelling into the right fracture branch. The different curves are for the different distances at which the particles were placed relative to the intersection in the left fracture branch (see Figure 1). 


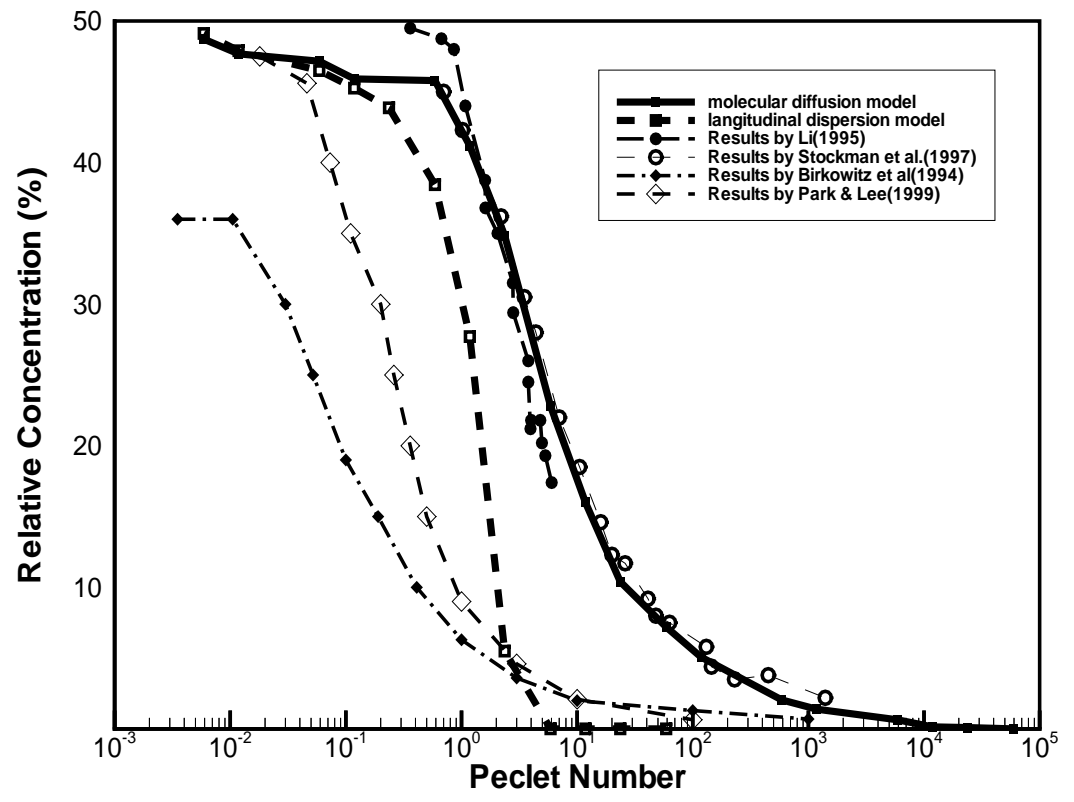

Figure 7. Comparisons of mixing characteristics (the percentage of the particles throughout the right-hand fracture branch) at the fracture intersection with an analytical result and two numerical results. 


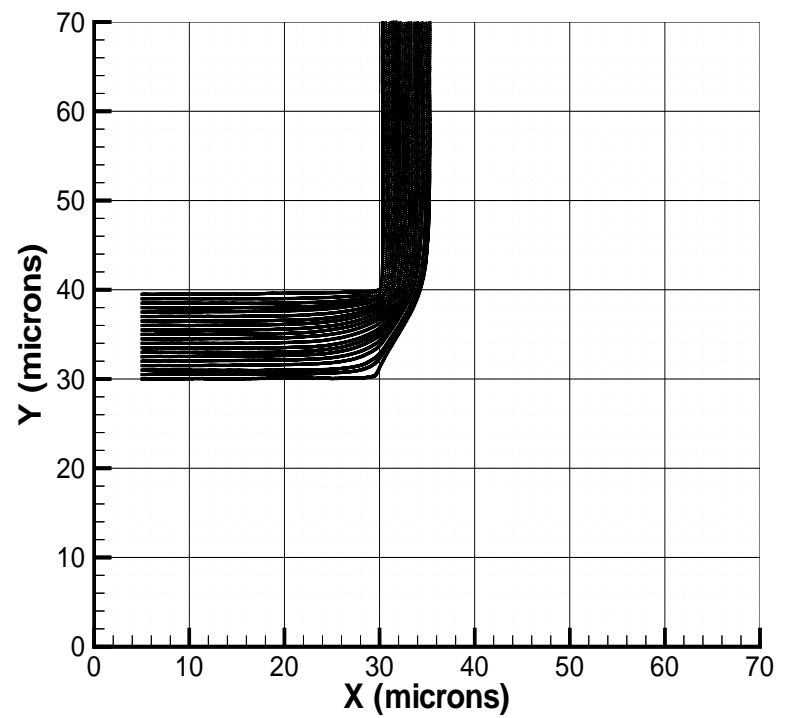

b

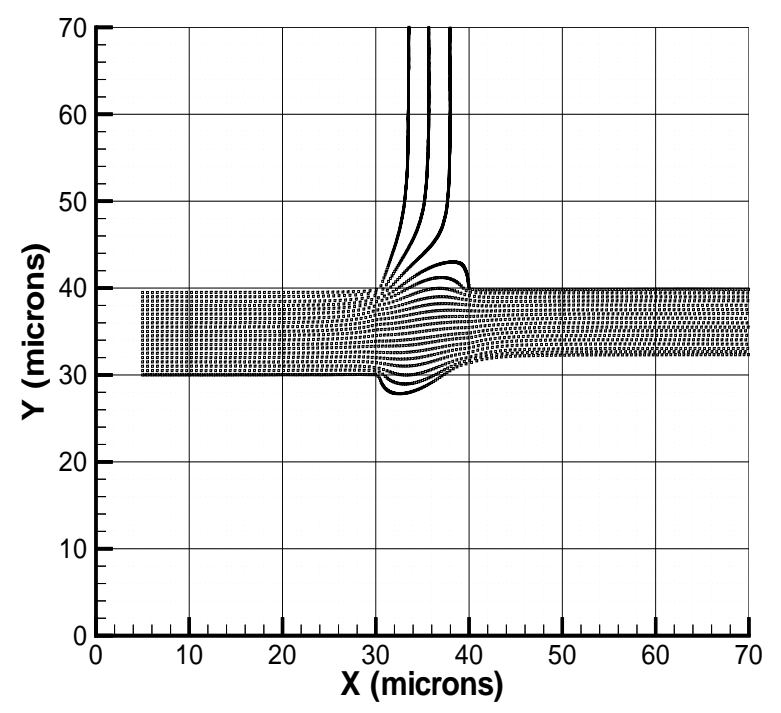

Figure 5. Spatial particle trace with Peclet number $1.18 \times 10^{5}$. (a) Model A: plug flow W35/S65 and (b) Model B: plug flow W83/S17. 


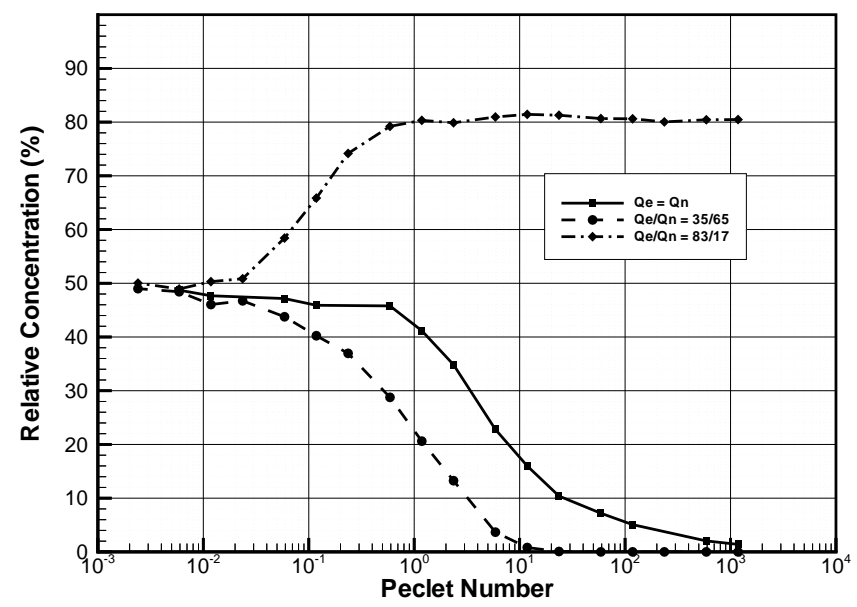

b

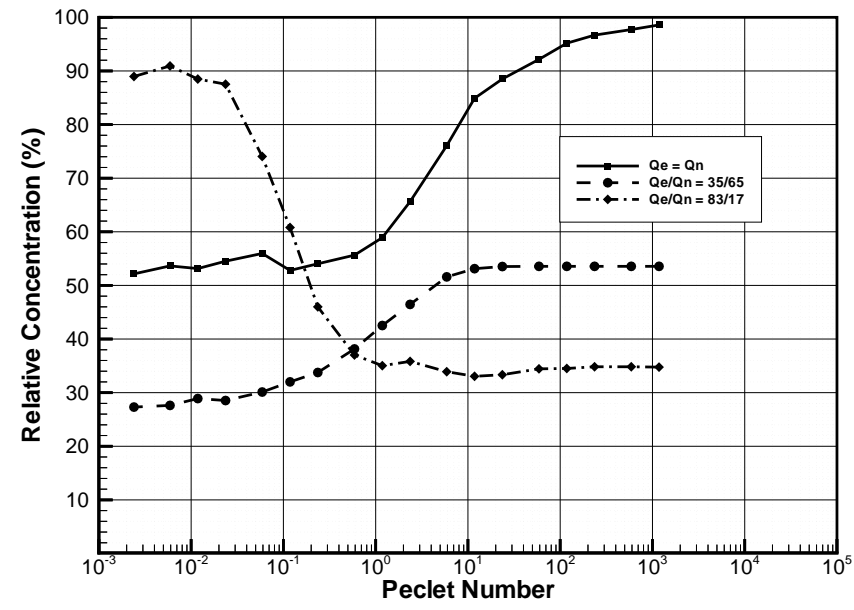

Figure 6. Comparisons of mixing characteristics at fracture intersections in Model A and Model B with the base model. (a) The relative concentration from the left fracture branch travelling into the right fracture branch. (b) The relative concentration from the left fracture branch travelling into the top fracture branch. 